\title{
Partitioning Hardware and Software for Reconfigurable Supercomputing Applications: A Case Study
}

\author{
Justin L. Tripp, Anders Å. Hanson, Maya Gokhale \\ Los Alamos National Laboratory \\ and \\ Henning Mortveit \\ Virginia Polytechnic Institute and State University
}

\begin{abstract}
Often reconfigurable systems are reported to have $10 \times$ to $100 \times$ speedup over that of a software system. However, the reconfigurable hardware must usually be combined with software to form an entire system. This system integration presents a hardware/software co-design problem with many system engineering issues. Here, we present traffic acceleration on the Cray XD1 supercomputer and describe the costs involved in different hardware/software trade-offs.

Categories and Subject Descriptors: Hardware [Performance and Reliability]: General

General Terms: Supercomputing, Integration

Additional Key Words and Phrases: Supercomputing, System Integration, HW/SW Co-design
\end{abstract}

\section{INTRODUCTION}

Reconfigurable supercomputing, the use of programmable logic to accelerate supercomputing platforms, has emerged as a novel approach to boosting performance. By porting compute and/or data intensive portions of an application to hardware, it has been conjectured that speedup of $10 \times$ to $100 \times$ might be achievable over conventional software systems, and in the past several years, reconfigurable supercomputers have been offered commercially. These systems (e.g.. SRC Computer, Cray, and Silicon Graphics) augment high performance microprocessors and interconnection networks with Field Programmable Gate Arrays (FPGAs), often combining a 3M-10M gate FPGA with each microprocessor node. Reconfigurable logic has been applied to supercomputing applications such as bioinformatics [TimeLogic 2004], simulation of physical phenomena, [Gokhale et al. 2004], and even high performance routing [Young et al. 2003].

Early experience with reconfigurable supercomputers suggests that, for suitable applications, FPGA implementations can indeed outperform equivalent generation microprocessors by factors of $10 \times$ to $100 \times$. When the data types are small fixed precision integers, such as in signal and image processing, customized, application-specific pipelines can be replicated on the FPGA, so that even with a clock rate that is in the 100 's of $\mathrm{MHz}$, the FPGA can easily perform a computation at orders of magnitude improvement over a microprocessor. In the newest generations of FPGAs, hundreds of hard multiply units on the chip offer the opportunity for even greater speedup in the integer domain, and competitive performance in single precision floating point.

Most studies performed to date on reconfigurable supercomputing applications focus on compute-intensive inner loop kernels, and report speedup over equivalent software. They do not consider the overall application performance which is determined by the fraction of the application that can be accelerated and the communication/synchronization costs. In order to achieve the highest level of performance on the overall application, each part of the system must be properly utilized. Costs associated with parallel processing

(C) 2005 Association for Computing Machinery. ACM acknowledges that this contribution was authored or co-authored by a contractor or affiliate of the U.S. Government. As such, the Government retains a nonexclusive, royalty-free right to publish or reproduce this article, or to allow others to do so, for Government purposes only.

SC | 05 November 12-18, 2005, Seattle, Washington, USA

(C) 2005 ACM 1-59593-061-2/05/0011 $\$ 5.00$ 
as well as hardware/software interaction must be carefully assessed to determine the best level of system integration.

By introducing FPGAs in a supercomputing configuration, opportunities exist for improving both coarsegrained (application-level) and fine-grained (instruction-level) parallelism. First, FPGAs provide a large amount of highly parallel, configurable hardware resources, which makes it possible to create structures, such as parallel multiply/add instructions, that greatly accelerate individual operations. Innermost loops can be unrolled to expose additional instruction level parallelism. Loops can also be accelerated through careful scheduling of compute and memory access instructions and by loop pipelining. At a higher level, these parallel structures can themselves be replicated to create additional levels of parallelism, up to the limit of the target device capacity.

To exploit the full capacity of FPGA-based computing, it is essential to carefully select the portion(s) of the code to implement in hardware. Ideally, the code kernel(s) should be able to exploit pipelining and replication, the sorts of spatial parallelism offered by FPGAs. This inner loop hardware acceleration can yield one to two orders of magnitude over software.

\subsection{Reconfigurable Supercomputing with Application to Roadway Traffic Simulation}

In this paper, we present a case study of porting a supercomputing application, road traffic simulation, onto a reconfigurable supercomputer, the Cray XD1. Our application, a simplified version of the TRANSIMS [Barrett et al. 2002] road network simulator, is part of the Los Alamos Infrastructure Simulation Suite. TRANSIMS has been used to perform detailed traffic simulations of large metropolitan areas.

Our choice of the TRANSIMS application for this experiment was based on the desire to fully utilize the FPGA's spatial parallelism. TRANSIMS uses the cellular automaton (CA) model, a highly parallel computational model. The theory of CAs has been well developed, and universal CAs capable of simulating the behavior of any Turing machine are known to exist [von Neumann 1963; Wolfram 2002]. The CA model for roadway traffic was proposed by Nagel and others [Nagel and Schreckenberg 1992; Nagel et al. 1995; Rickert et al. 1996]. Thus the road traffic CA has the potential to effectively use the FPGA's spatial parallelism. In addition, this application uses small integer data types and simple arithmetic operations that are easily expressed as hardware circuits.

However, the amount of speedup actually realized also depends on how well the problem can fit on the available hardware resources, often a single FPGA and a few MB of memory per node. TRANSIMS is truly a supercomputing application. The large metropolitan road networks simulated by this tool encompass $6-60 \mathrm{M}$ road cells. Thus our study also assesses how well the Cray XD1 configuration fits the TRANSIMS data sets.

Starting from application profiling to determine the compute-intensive portions, we describe our experience with partitioning the application between software and hardware, creating the hardware design, optimizing communication, and identifying other system integration issues. Our work helps determine desirable characteristics for next generation reconfigurable supercomputers.

\subsection{Related Work}

A wide variety of reconfigurable supercomputing applications have been reported over the past several years. Particularly impressive results have been reported for signal and image processing, cryptography, bioinformatics, and network security. [Gokhale and Graham 2005] provides a comprehensive survey of FPGA implementations in these application areas.

More directly related to this paper, previous work has been reported on cellular automata mapped onto programmable hardware. Recently, Halbach and Hoffman implemented a CA model in FPGA logic [Halbach and Hoffmann 2004]. On an experimental low-end platform, they obtained $3 \times$ and $22 \times$ speedup for a CA

rule of medium and high complexity, respectively. In their paper, they also claimed that several thousand times speedup could potentially be achieved compared to a pure software solution, simply using the latest high-end FPGA technology.

In the Halback and Hoffman study, the speedup of an FPGA system is measured by comparing raw speed of the FPGA to that of a pure software implementation. Most useful systems need to combine compute-intensive kernels in the FPGAs with a control and coordination application running in software. The overall system speedup is not usually reported. The accelerated computation on the FPGA is not the only component and the overall speedup is controlled by Amdahl's Law. 
An example of the gap between kernel speedup and application speedup is demonstrated by the integration of Triple-DES acceleration into Virtual Private Network (VPN) communication using the Pilchard FPGA accelerator [Cheung and Leong 2002]. Their FPGA implementation of Triple-DES is able to encrypt data at a rate three times that of software. However, with software and hardware integrated, the system speedup was only $1.3 \times$ for TCP and $1.16 \times$ for UDP over the pure software version. This is due to: (i) only $50 \%$ of the software time is spent in Triple-DES, and (ii) encryption is done on a packet-by-packet basis. The FPGA system is not faster than software for small sized packets, likely due to data transfer overheads. Network traffic, however, often contains smaller packets which reduce the performance gains even further.

Not all systems suffer from the costs of data transfer overhead. An FPGA-based acceleration of computation electromagnetics with millions of data nodes is able to achieve three times speed over a 30 node PC cluster[Durbano et al. 2004]. In this case, the system can operate on up to 256 million nodes and process 30 million nodes/sec. Ninety PCs are required to achieve the same rate and cost considerably more than the single FPGA board. This system was able to achieve high speed since: (i) all processing occurred on the FPGA and data transfer was minimal, and (ii) the FPGA board had 4 banks of 4 Gigabyte DDR SRAM which provided $8.5 \mathrm{~GB} / \mathrm{s}$ of data bandwidth. These combined for this application to provide a considerable performance gain.

\section{ACCELERATION OF TRANSIMS}

TRANSIMS was originally developed to run on a Linux cluster due to the scale of the urban traffic simulation. As an example of the problem size, the Portland road network representation has roughly 6.6 million road cells. For cities like Chicago, Houston, and Los Angeles this number is larger by a factor of $3 \times$ to $10 \times$.

In order to explore FPGA-based hardware acceleration of the TRANSIMS micro-simulator, we first profiled the execution time of a single-processor, pure software implementation. Targeting the Cray XD1 supercomputer, we profiled our executable on an AMD Opteron 200 series processor with 8 Gigabytes of memory. The results are listed in the first column of Table I below. (Columns two and three will be discussed in Section 4.)

Table I. Execution Time Profile of one Simulation Step.

\begin{tabular}{|l|r|r|r|}
\hline \hline Activity & $\begin{array}{r}\text { Fraction } \\
\text { w/o Comm. }\end{array}$ & $\begin{array}{r}\text { Fraction } \\
\text { w/o Push }\end{array}$ & $\begin{array}{r}\text { Fraction } \\
\text { w/ Push }\end{array}$ \\
\hline Software to hardware & - & $0.2 \%$ & $0.2 \%$ \\
Lane change update & $16.8 \%$ & $14.5 \%$ & $18.6 \%$ \\
Velocity update & $27.6 \%$ & $23.9 \%$ & $28.3 \%$ \\
Position update & $37.9 \%$ & $32.7 \%$ & $37.4 \%$ \\
Intersection update & $17.7 \%$ & $15.3 \%$ & $15.3 \%$ \\
Hardware to software & - & $13.4 \%$ & $0.2 \%$ \\
\hline \hline
\end{tabular}

From the profile, we understand that one could potentially achieve $5 \times$ speedup by processing (i) lane change updates, (ii) velocity updates, and (iii) position updates in configurable logic, such as FPGAs. This strategy has the additional advantage that all hardware processing would be governed by a simple, homogeneous set of rules (in contrast to the more complicated set of rules that control intersections). If a single FPGA could serve two Opteron processors, the XD1 platform with its twelve Opteron processors and six FPGAs would then be able to achieve $60 \times$ speedup compared to a single Opteron. Certainly, this is a rough upper bound as we have not accounted for communication costs, but it is nonetheless a number that can serve as motivation for our approach.

In the following, we will briefly describe a scalable hardware design, and we will then discuss the associated integration costs. Before doing so, we first need to describe the problem in somewhat more detail.

$\mathrm{k}$

\subsection{Cellular Automaton Traffic Modeling}

The TRANSIMS road network simulator, which is based on [Nagel and Schreckenberg 1992; Nagel et al. 1995; Rickert et al. 1996], can best be described as a cellular automaton computation on a semi-regular grid 
or cell network: The representation of the city road network is split into nodes and links. Nodes correspond to locations where there is a change in the road network such as an intersection or a lane merging point. Nodes are connected by links that consist of one or more unidirectional lanes. A lane is divided into road cells each of which are 7.5 meters long. One cell can hold at most one car, and a car can travel with velocity $v \in\{0,1,2,3,4,5\}$ cells per iteration step. The positions of the cars are updated once every iteration step using a synchronous update, and each iteration step advances the global time by one second. The basic driving rules for multi-lane traffic in TRANSIMS can be described by a four-step algorithm. In each step we consider a single cell $i$ in a given lane and link. Note that our model allows passing on the left and the right. To avoid cars merging into the same lane, cars may only change lanes to the left on odd time steps and to the right on even time steps. This convention, along with the four algorithm steps described below, produces realistic traffic flows as demonstrated by TRANSIMS.

Local Driving Rules. The micro-simulator has four basic driving rules. We let $\Delta(i)$ and $\delta(i)$ denote the cell gap in front of cell $i$ and behind cell $i$, respectively. $v$ is the velocity of the car in cell $i$ and $v_{\text {max }}(i)$ is the maximum velocity for this particular road cell, which may be lower than the global $v_{\max }$ (e.g., a local speed limit).

(1) Lane Change Decision: Odd time step $t$ : If cell $i$ has a car and a left lane change is desirable (car can go faster in target lane) and permissible (there is space for a safe lane change) flag the car/cell for a left lane change. The case of even numbered time steps is analogous. If the cell is empty nothing is done.

(2) Lane Change: Odd time step $t$ : If there is a car in cell $i$, and this car is flagged for a left lane change then clear cell $i$. Otherwise, if there is no car in cell $i$ and if the right neighbor of cell $i$ is flagged for a left lane change then move the car from the neighbor cell to cell $i$. The case of even time steps $t$ is analogous.

(3) Velocity Update: Each cell $i$ that has a car updates that car's velocity using the two-step sequence:

$-v_{\text {next }}:=\min \left(v+1, v_{\max }(i), \Delta(i)\right)$ (acceleration)

-If $\left[\right.$ UniformRandom ()$\left.<p_{\text {break }}\right]$ and $[v>0]$ then $v_{\text {next }}:=v-1$ (stochastic deceleration).

(4) Position Update: If there is a car in cell $i$ with velocity $v=0$, do nothing. If cell $i$ has a car with $v>0$ then clear cell $i$. Else, if there is a car $\delta(i)+1$ cells behind cell $i$ and the velocity of this car is $\delta(i)+1$ then move this car to cell $i$. The nature of the previous velocity update pass guarantees that there will be no collisions.

All cells in a road network are updated simultaneously. The steps 1-4 are performed for each road cell in the sequence they appear. Each step above is thus a classical cellular automaton $\Phi_{i}$. The whole combined update pass is a product CA, $\Phi=\Phi_{4} \circ \Phi_{3} \circ \Phi_{2} \circ \Phi_{1}$, i.e., a functional composition of classical CAs. Note that the CAs used for the lane change and the velocity update are stochastic CAs. The rationale for having stochastic braking is that it produces more realistic traffic. The fact that lane changes are done with a certain probability avoids slamming behavior where whole rows of cars change lanes in complete synchrony.

Intersections and Global Behavior. The four basic rules handle the case of straight roadways. TRANSIMS uses travel routes to generate realistic traffic from a global point of view. Each traveler or car is assigned a route that he/she has to follow. Routes mainly affect the dynamics near turn-lanes and before intersections as cars need to get into a lane that will allow them to perform the desired turns. To incorporate routes the road links need to have IDs assigned to them. Moreover, to keep computations as local as possible, cells need to hold information about the IDs of upcoming left and right turns. The details of the intersection rules are explained in [Gokhale et al. 2005].

\subsection{Hardware Acceleration of Traffic Simulation}

FPGAs provide the highest level of parallelism available in programmable hardware. The fastest way to simulate traffic would be to structurally build the entire traffic network in the FPGA fabric. However, despite the large amount of available hardware, it would take approximately 12,400 FPGAs to implement the entire Portland network. Despite the large amount of parallelism, the structural approach is prohibitively expensive. 
A common approach used in many FPGA applications (e.g., signal processing, encryption, etc.) is to stream data through a number of computational units. In the context of traffic simulation, streaming can be achieved through a computation engine that processes a stream of road data and subsequently outputs a stream of updated data. Using this approach, the number of road cells are not limited to available FPGA area. A streaming hardware design is scalable and can handle large-scale road networks.

In our streaming design, we partition the road network in such a way that straight road sections are processed by the hardware, while intersections and merging nodes are updated by a software module. Most importantly, this hybrid hardware/software strategy means that hardware processing is governed by a simple, homogeneous set of traffic rules, while all road plan decisions are handled by software.

The data representing straight lanes is fed to the hardware update engine against the flow of traffic, starting from the end of each lane. However, due to the partitioning of the road network, the cars in the last $v_{\max }$ cells of each lane cannot be updated since the engine lacks knowledge about the network topology and the road plans. For this reason we define an overlap region I (see Figure 1(a)), which is the last $v_{\max }$ cells of each lane, and because of the processing direction of the computation engine, these cells are processed first. Although cars that are inside an overlap region at the beginning of the hardware computation cannot be updated by the engine, it is important to note that the engine can move other cars into these first cells during its computational pass. Naturally, the software module needs to update the position and velocity of cars inside the overlap regions at the end of each hardware update pass.

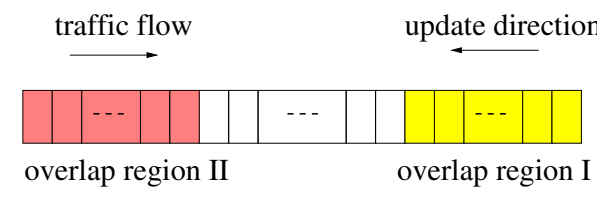

(a) Road Link Structure

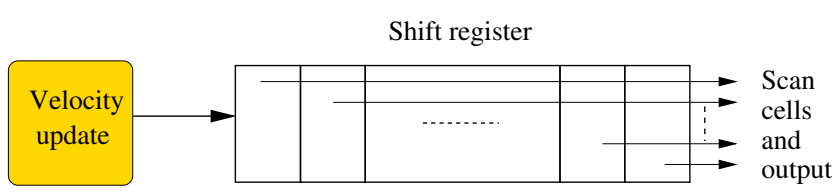

(b) Position Update

Fig. 1. Traffic Acceleration Road Link Structure and the Position Update Hardware

The software must also write information to the first $v_{\max }$ cells of each lane, which corresponds to new cars moving into a lane (arriving from other lanes, either through an intersection or by merging). However, computing velocities and positions of these new cars requires complete knowledge of the first $v_{\max }$ cells of each lane. Therefore, the first $v_{\max }$ cells of each lane constitute another overlap region (see overlap region II in Figure 1(a)) whose state information must be known in software.

In consequence of the hardware/software partitioning, there is a need for synchronizing the status of road cells in the overlap regions. In order to minimize the cost of memory synchronization, it is desirable to process only single-lane traffic in hardware (and let software process multiple-lane traffic). The reason is that multiple-lane traffic would require the synchronization of more than $v_{\max }$ cells since lane change permissibility assumes knowledge of preceding cells. ${ }^{1}$ Restricting the hardware computation to single-lane traffic may sound like a severe limitation. However, the road lane distribution for Portland, shown in Figure 2, reveals that $90 \%$ of all roads are one-lane roads. This means that an overwhelming majority of all road cells will in fact be updated in hardware, and significant hardware acceleration remains a possibility.

The streaming engine calculates a car's position by shifting the car one cell every clock cycle (see Figure 1(b)) until its newly calculated velocity matches the distance from the end of a shift register, which is $v_{\max }+1$ cells long. At the point when there is a match, the change state block exports all car information to the destination road cell. This pipelining design makes it possible for the computation engine to read and write one word of road data every clock cycle. If we have access to $N$ concurrent data streams, it would be advantageous to instantiate $N$ parallel replicas of the compute engine.

\footnotetext{
${ }^{1}$ There is an exception: If the cells were empty at the beginning of the update pass, the software module does not need to read back the updated status since hardware can only move cars from the first cells.
} 


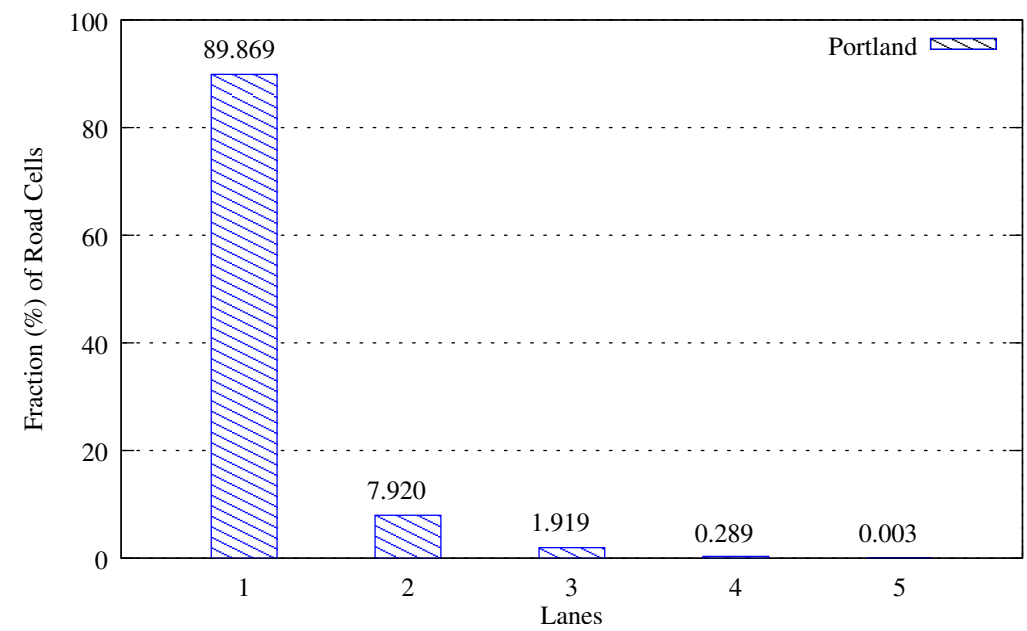

Fig. 2. Road Lane Distribution for the Portland Data

\section{THE CRAY XD1 RECONFIGURABLE SUPERCOMPUTER}

The Cray XD1 supercomputer combines high performance microprocessors, a high speed, low latency interconnect network, and reconfigurable computing elements. This provides a system where data transfer latencies and bandwidth associated with I/O buses is greatly reduced. Normally, reconfigurable systems need to process large amounts of data to amortize the cost of transferring the data to the FPGA. However, with tight integration of processors and reconfigurable computing, a supercomputing problem can be split between the CPU and FPGA with close synchronization and fast communication between software and hardware.

A single chassis of the Cray XD1, consists of 12 AMD Opteron 200 series processors, with up to 8 Gigabytes of memory per processor. The processors are paired in SMP processor modules as shown in Figure 3(a). Each processor has 1 or 2 Cray RapidArray links that connect to a fully non-blocking Cray RapidArray fabric switch. The switch provides either $48 \mathrm{~GB} / \mathrm{s}$ or $96 \mathrm{~GB} / \mathrm{s}$ total bandwidth between the SMP pairs. The RapidArray fabric switch is able to achieve $1.8 \mu$ s MPI latency between SMPs.

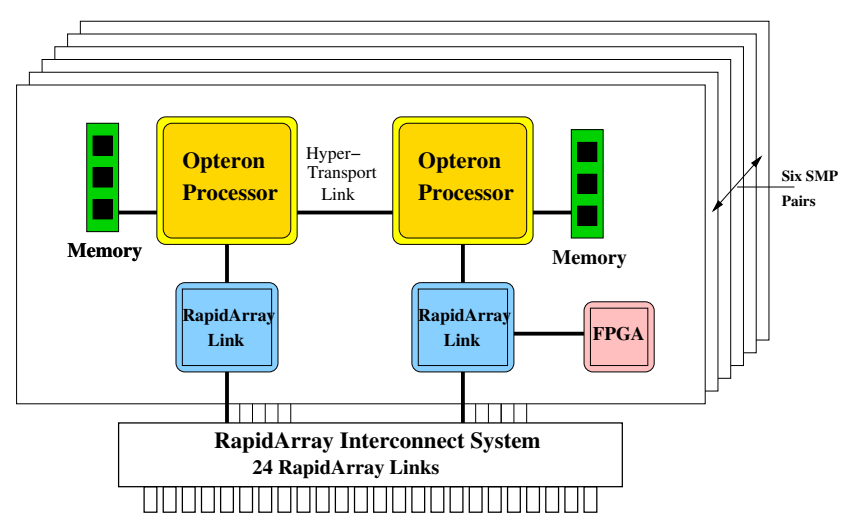

(a) Processor Module

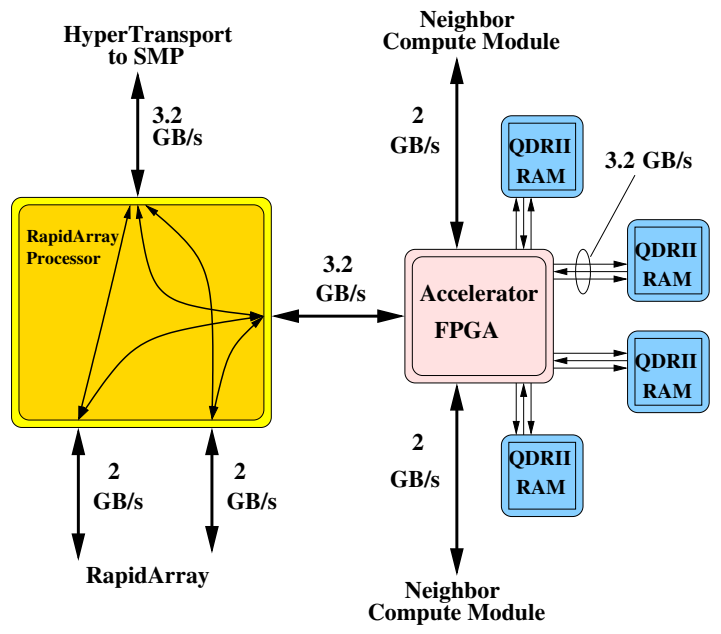

(b) FPGA Module

Fig. 3. Structure of the Cray XD1 
As shown in Figure 3(b), one Xilinx Virtex-II Pro (30 or 50) is available for each processor module from the RapidArray fabric. An FPGA has $3.2 \mathrm{~GB} / \mathrm{s}$ link to the RapidArray fabric, which connects to the local processors or to other processors on the fabric. The FPGA also has dedicated $2 \mathrm{~GB} / \mathrm{s}$ RocketIO links to the neighboring SMP module in the same chassis. Four QDR SRAMs are connected to each FPGA providing $3.2 \mathrm{~GB} / \mathrm{s}$ of bandwidth at $200 \mathrm{MHz}$ [Cray, Inc. 2004a].

System development is performed by first manually partitioning the software and hardware for the reconfigurable system. The FPGA design is written in a Hardware Description Language (HDL) like VHDL or Verilog. This design is combined with behavioral system cores that help simulate the entire system operation. Once the system has been verified in simulation, the design is then synthesized into a form that can be used to program the FPGAs. With the FPGA programmed, the software can then be integrated with the FPGA hardware.

On the reconfigurable system, the Opteron SMP modules run Linux, and MPI is provided to communicate to other Opteron modules. The FPGAs are accessed under Linux using device drivers provided by Cray. Cray's FPGA API provides functions for loading, resetting, and executing FPGA designs. Functions are also provided for mapping the FPGA's memory into the operating system's memory space and for accessing registers defined on the FPGA.

As described in the XD1 FPGA Development manual [Cray, Inc. 2004b], there are asymmetric costs associated with reads and writes between the host and the FPGA's QDR SRAMs. Writes can take advantage of write combining in the Linux kernel and are non-blocking. On the other hand, reads are blocking and cannot be combined. This creates an asymmetric cost which must be overcome.

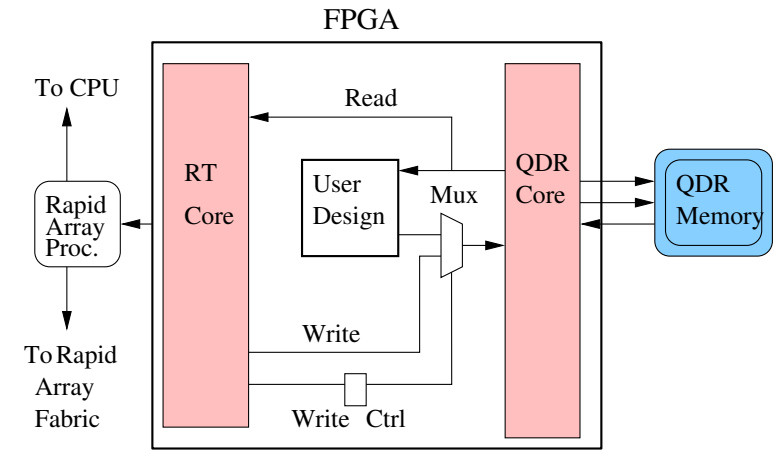

(a) FPGA SRAM access

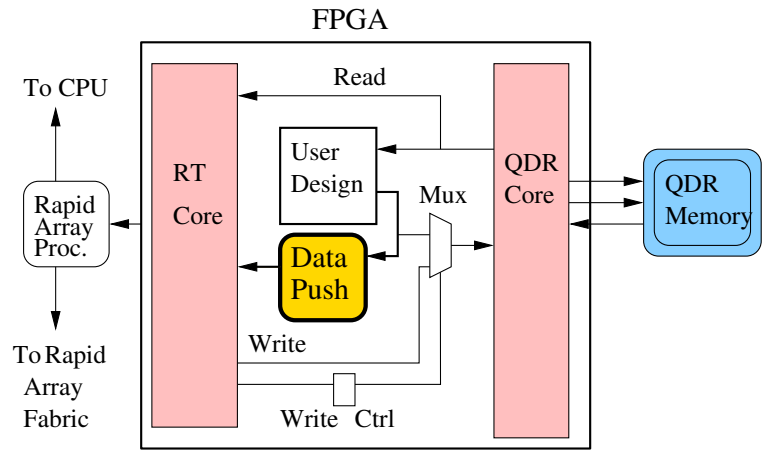

(b) FPGA Write-Only Architecture

Fig. 4. FPGA QDR SRAM access (only one memory shown). Figure 4(a) shows the provided approach for memory access. Figure 4(b) shows the data push process added to make the design write-only.

The method for accessing the FPGA's SRAMs is shown in Figure 4(a). The multiplexor (mux) between the traffic engine and the RapidArray Transport (RT) core allows both the host and the traffic engine to write to the SRAMs. Reads do not require a mux for the data, but both read and write require muxes for the address lines (not shown). Using this setup we benchmarked reads and writes from the host to the FPGA.

Table II. Read and Write Bandwidth (MB/s) for Hosts to QDR SRAMS

\begin{tabular}{|l|r|r|r|}
\hline \hline & Array & Pointer & Memcpy \\
\hline Read & 5.94 & 5.95 & 6.01 \\
Write & 1260 & 1320 & 1320 \\
\hline \hline
\end{tabular}

Table II shows the bandwidth results for reads and writes accomplished using three different approaches. The FPGA SRAMs are mapped into the hosts local memory and can be accessed using arrays with indexes, 
using pointers, or by using the memcpy function call. Array accesses were found to be slight slower, but the bandwidth is roughly equivalent. However, the difference in cost between reads and writes is a factor of 200 . This asymmetric cost is overcome by using a "write-only" architecture as suggested by Cray. The host must write data to the FPGA memory and the FPGA must write the data that the host needs to read.

Figure 4(b) details the hardware added to a user design to make it a write-only architecture. A separate data push process is added to update shared memory on the host as well as the FPGAs local memory. Using the data push process allows the user design to reduce the time required to transmit data updates between the FPGA and the host. This allows more time on the processor and FPGA to be spent processing data instead of communicating.

\section{SOFTWARE/HARDWARE COMMUNICATION COSTS}

Using the data streaming approach, data must be streamed through the compute engine to calculate the traffic update. There are two different approaches that can be used to move the data to the engine. As described in Section 1.2, the Pilchard FPGA board had data streaming into the FPGA and back to main memory when it was encrypted. On the other hand, the computational electromagnetics engine streamed data between memories attached to the FPGA. This local memory traffic allowed their application to achieve high levels of performance, since they were not limited by the rate at which data could move back the main memory of the system. Both of these approaches have different trade-offs, since the Pilchard approach allows unbounded data to be processed continuously. The eletromagnetics engine can only operate on data sets that fit in its 16 GBytes of memory.

Since the hardware kernel of the traffic application does not need full visibility on the host, we chose to use a local streaming approach similar to that of the electromagnetics engine. Each of the four memories on a Cray XD1 FPGA node was paired with a compute engine calculating new traffic results. These results can either be read out of the FPGA's memories by the host or pushed back to the host by the FPGA.

Once the FPGA design is tested and completed, it must be integrated with the software and the entire system speed evaluated. Software integration consists of two parts: communication with the FPGA, and preparing data for transfer to the FPGA. This software integration and the speed of the entire system must be measured to understand the cost of using the FPGA.

The results from profiling the execution time, taking the cost of memory synchronization into account, are presented in columns two and three of Table I. Column two represents the results when the data is read by the host. The results reflect the impact of the slow transfer of data from hardware to software when the host is instigating the read. The third column shows how communication is reduced to nearly nothing by overlapping the data transfer and the computation.

Table III compares the processing rates for (i) the raw FPGA without considering communication, (ii) local streaming using the host to control communication, (iii) an estimated amount for an external streaming approach, and (iv) the local streaming approach pushing back the partial data to the host machine. If results calculated by the FPGA are read directly by the host, the speedup is only $4.5 \times$. This is due to the blocking nature of reads from the host. Pushing the results up to the host results in a more impressive speedup of $34.4 \times$.

Table III. Comparison of Streaming including Communication Costs. External Stream is estimated based on data transfer rates using the push and transferring the maximum number of single lane road segments.

\begin{tabular}{|l|r|r|r|r|r|}
\hline \hline & $\begin{array}{r}\text { No comm. } \\
\text { V2p50 }\end{array}$ & $\begin{array}{r}\text { w/o Push } \\
\text { V2p50 }\end{array}$ & $\begin{array}{r}\text { Ext. Stream } \\
\text { V2p50 }\end{array}$ & $\begin{array}{r}\text { Push } \\
\text { V2p50 }\end{array}$ & $\begin{array}{r}2.2 \mathrm{GHz} \\
\text { Opteron }\end{array}$ \\
\hline Cells/sec & $7.2 \times 10^{8}$ & $2.56 \times 10^{7}$ & $4.22 \times 10^{7}$ & $1.96 \times 10^{8}$ & $5.7 \times 10^{6}$ \\
\hline Speedup & 126.3 & 4.5 & 7.4 & 34.4 & 1.0 \\
\hline \hline
\end{tabular}

It should be emphasized, however, that $34.4 \times$ refers to the speedup of processing straight lanes. The overall speedup is limited to $5 \times$ as seen from Table I, but this assumes that we have enough memory resources to fit all straight road cells. Since only about 2 million road cells can fit in the QDR memory, the overall system speedup becomes $11 \%$. 


\section{DISCUSSION}

Partioning Hardware and Software for Reconfigurable Supercomputing Applications: A Case Study

FPGAs are normally used for applications that are more compute intensive than data intensive, such as DSP algorithms like the FFT. Clearly, this picture changes as soon as we try to hardware-accelerate more typical high-performance computing algorithms. The integration of software and hardware has costs that must be well understood in order for the entire system to realize an overall speedup. There are two main approaches that can solve the performance bottlenecks in the traffic simulation acceleration. First, the algorithmic implementation can be optimized to realize a better use the hardware and software. Second, the reconfigurable system could be improved to better support the traffic simulation problem.

\subsection{Algorithmic Optimization}

Three different approaches may be used to optimize the algorithmic approach to traffic simulation. First, it is possible to use a more succinct representation of the traffic simulation road cells. Second, we could use more compute nodes of the Cray XD1. Third, the development tools can improved to reduce the cost of design exploration.

A straightforward (non-optimized) road cell representation uses 64 bits, which is the full width of the SRAMs. $^{2}$ Now, the depth of the memories prove to be a bottleneck as only about 2 million cells can be off-loaded to hardware (recall that the Portland road network model has about 6.6 million cells). Twice as many cells (i.e. 4 million) could easily have been processed by the FPGA, but the memory limitation means that they have to remain at the host.

Recall that the data passes through the FPGA on its way to the QDR memory, as seen from Figure 4(b). One option would be to compress the road data prior to storing it, and uncompress the data before it is being fed to the compute engine. A simple on-line codec algorithm would easily fit on the FPGA since the traffic design currently requires $30 \%$ of the chip resources (a single compute engine occupies less than $4 \%$, while the RT/QDR cores occupy about 15\%). For example, run-length encoding could be employed. We can also imagine more aggressive compression techniques that exploit the semantics of the traffic data.

For our application, we could also devise a more succinct road cell representation - at the cost of increased software complexity. As already explained, the CPU is responsible for putting new cars in each road segment (corresponding to cars coming from adjacent segments). Since only single-lane traffic is off-loaded to the FPGA, we would not have to encode and transfer any car IDs. Instead, the CPU could keep track of the order in which these new cars enter each segment, and cars obviously have to leave a single-lane road segment in the order in which they enter.

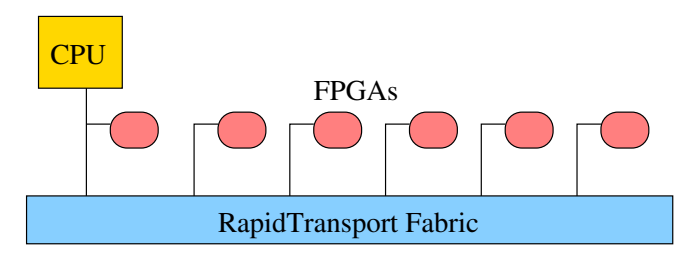

(a) One CPU and many FPGAs

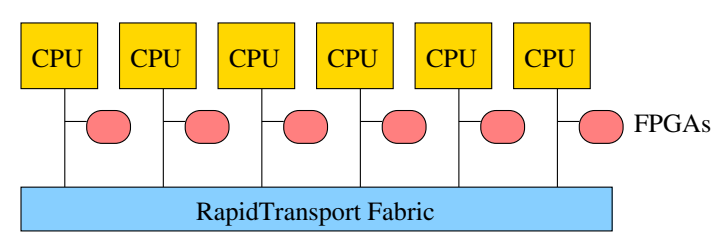

(b) Many CPUs and many FPGAs

Fig. 5. Approaches for increasing Parallelism

Since the XD1 platform has six compute nodes, each having two Opterons plus one FPGA with its associated QDR memory, we could potentially use all six nodes in our design. We then have two possibilities: (i) we can divide the full problem so that each node is updating straight road sections (processed by the FPGAs) as well as intersections (processed by the Opterons) as shown in Figure 5(b), or (ii) we could divide the straight road sections between the FPGAs while a single Opteron (see Figure 5(a)) is updating all intersections. Alternative (ii) is interesting because of its simplicity as only ghost processes are running on

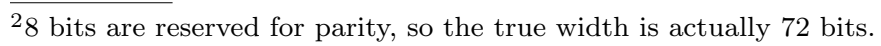


five of the hosts, whose sole responsibility is to control the FPGAs (i.e., start the FPGAs, poll them to see if they are done, and send back the data to the mother CPU, which is processing all intersections). To fully divide the problem is of course more complicated since it would require the road network to be partitioned across intersections. Also, synchronization between the software modules, as well as between the software modules and the hardware becomes more involved (compared with alternative (ii) in which a single software module needs to synchronize with the hardware).

The two previous strategies rely on MPI, which is the communication method provided by Cray. It might also be possible for the FPGAs to communicate directly across the RT fabric, but there is currently no API that supports this. Note that this approach is desirable since the communication overhead is very small in contrast to the overhead associated with MPI.

Since there are many FPGAs in the Cray XD1, it should be possible for one CPU to send data to many of them. Currently this is not the case, but it may not be that difficult to implement. Using 2 FPGAs (and their memories) would have allowed the system to handle all of the Portland traffic example. Adding additional FPGAs would easily allow systems three times the size of Portland. Using this approach would allow easy scalability without changing how the FPGAs are used. Also it is conceivable for problems to require additional computation on the FPGA. Allowing easy FPGA-to-FPGA communication would aid in this task.

A major hindrances to rapid application development with reconfigurable hardware are the tools that are provided to accomplish this task. Typically reconfigurable hardware development requires an hardware engineer familiar with FPGA development. The hardware design is written VHDL or Verilog and simulated using a hardware description language (HDL) simulator. Simulation normally involves using models for external connections to memories and the rest of the system. These models are usually an abstraction of the behavior found the in the rest of the system due to high cost of true simulation. After simulation of a design has been verified, then the design must be converted into a form that can be understood by the reconfigurable hardware. This involves a set of synthesis tools that transform the HDL description in to a description ("bitstream") that may be downloaded onto the reconfigurable hardware. Unfortunately, this process involves several difficult optimization problems (e.g., function mapping, wire routing, etc.) Normally, it can take one or more hours to generate the bitstream. The entire process, simulation and synthesis must be completed for every change required in the hardware design.

The difficult task described above seriously impacts the ability of a designer to perform design exploration. Every small change in a design results in a several hour wait to test the new hardware. Reducing the time to synthesize designs would allow for more design exploration and better designs. Automated tools are also needed to help in aid in the HW/SW co-design process. These tools need to help a designer understand the trade-offs between data communication and computation on the FPGA.

\subsection{System Architecture Improvements}

Several approaches could be used to create the next generation of reconfigurable hardware. Improvements would include deeper memories, more memories, a mixture of memory types and high speed FPGA-to-FPGA communication. These improvements will make the next generation reconfigurable hardware able to handle more difficult computation.

In almost all cases memory is expensive and developers would like more memory. In the case of the TRANSIMS data sets, they can grow much larger than the current Portland example. Keeping the data close to the FPGA reduces the amount of time spent transferring data and increases the amount of time in which data can be processed. Larger memories would increase the amount of data processed by the FPGA.

The traffic design is limited to four traffic engines since there are only four memory ports available for use by the FPGA. This small number of ports restricts the amount of parallelism that can be achieved on the FPGA. Since each traffic engine requires only $4 \%$ of the total FPGA area, it is feasible that up to 20 engines could operate concurrently. This would take advantage of the large amount of parallelism available in traffic simulation.

Since large SRAM banks are expensive, another approach to provide large memory would be to include a DRAM bank on the FPGA. The DRAM bank would allow the FPGA to access hundreds of megabytes of memory instead of just tens. The TRANSIMS data was accessed in a linear fashion and could have easily 
used DRAM instead of SRAM. However, the electromagnetic computation example used both since DRAM is not as efficient at random access as SRAM. The SRAM was used as a quick random access cache.

The Virtex2pro FPGAs found in the Cray XD1 have several fast gigabit serial transceivers that can be used to transmit data. These could be used to connect the FPGAs using their own communication network. This would allow the FPGAs to be combined and to communicate without competing for the bandwidth on the RapidArray Fabric. In the traffic example, this could allow a multi-FPGA design to easily communicate boundary information.

\section{CONCLUSIONS}

In this paper, we have addressed some of the integration aspects related to hardware/software co-design. These issues are often ignored when hardware acceleration of software implementations are reported, because need for synchronization is often overlooked. We investigated a cellular automaton model for simulating largescale urban roadway traffic and used a Cray XD1 as our target platform. Based on the execution profile of a pure software implementation, and based on the problem structure, we chose to partition the simulation such that all straight road sections are processed in reconfigurable hardware, while the intersections are processed by the CPU.

Disregarding communication costs, the hardware would achieve $126 \times$ speedup over software. This number reflects the achievable processing gain for straight road segments, but it is inaccurate in that it does not take the cost of communication overhead into account. If the communication cost is included, the speedup drops to $34 \times$ - still a respectable number, which was only obtained by letting the FPGA push back data to the host (instead of having the host read the FPGA memories). However, $34 \times$ only refers to the speedup of single-lane road sections. The overall speedup is of course limited by Amdahl's law, and a factor of 5 would be possible if the entire dataset could fit in the SRAM memory banks. We discussed alternative methods to achieving the potential $5 \times$ speedup such as data compression, increasing the number of FPGA nodes used, and increasing the size of the memories. Our work demonstrates that partitioning and system integration issues play a crucial role in realizable speed up on reconfigurable supercomputers.

\section{REFERENCES}

Barrett, C. L., Beckman, R. J., Berkbigler, K. P., Bisset, K. R., Bush, B. W., Campbell, K., Eubank, S., Henson, K. M., Hurford, J. M., Kubicek, D. A., Marathe, M. V., Romero, P. R., Smith, J. P., Smith, L. L., Stretz, P. E., Thayer, G. L., Van Eeckhout, E., and Williams, M. D. 2002. TRansportation ANalysis SIMulation system (TRANSIMS) portland study reports.

Cheung, O. Y. H. And Leong, P. H. W. 2002. Implementation of an fpga based accelerator for virtual private networks. In Field-Programmable Technology, FPT Proceedings IEEE Internation Conference on. IEEE, 34-41.

Cray, Inc. 2004a. Cray XD1 Datasheet. Cray, Inc., Seattle, WA.

Cray, Inc. 2004b. Cray XD1 FPGA Development. Cray, Inc., Seattle, WA.

Durbano, J. P., Ortiz, F. E., Humphrey, J. R., Rsen F. Curt, P., And Prather, D. W. 2004. FPGA-based acceleration of 3D finite-difference time-domain metho d. In Proceedings of the IEEE Symposium on Field-Programmable Custom Computing Machines. Napa, CA.

Gokhale, M., Frigo, J., Ahrens, C., Tripp, J. L., And Minnich, R. 2004. Monte carlo radiative heat transfer simulation on a reconfigurable computer. In International Conference on Field Programmable Logic and Applications.

Gokhale, M., Hansson, A., Mortveit, H., And Tripp, J. 2005. Urban traffic simulation modeling for reconfigurable hardware. In Industrial Simulation Conference. Berlin, Germany.

Gokhale, M. B. and Graham, P. S. 2005. Reconfigurable Computing: Accelerating Computation with Field-Programmabl e Gate Arrays. Springer Verlag.

Halbach, M. And Hoffmann, R. 2004. Implementing cellular automata in FPGA logic. In 18th International Parallel and Distributed Processing Symposium. Santa Fe, NM, 3531-3535.

Nagel, K. And Schreckenberg, M. 1992. A cellular automaton model for freeway traffic. Journal de Physique I 2, $2221-2229$.

Nagel, K., Schreckenberg, M., Schadschneider, A., And Ito, N. 1995. Discrete stochastic models for traffic flow. Physical Review E 51, 2939-2949.

Rickert, M., Nagel, K., Schreckenberg, M., And Latour, A. 1996. Two lane traffic simulations using cellular automata. Physica A 231, 534-550.

TimeLogic. 2004. Timelogic corporate website. http://www.timelogic.com.

Tripp, J. L., Mortveit, H. S., Nassr, M. S., Hansson, A. A., And Gokhale, M. 2004. Acceleration of traffic simulation on reconfigurable hardware. Tech. Rep. LA-UR 04-2795, Los Alamos National Laboratory, Los Alamos, NM. 


\section{Tripp et al.}

von Neumann, J. 1963. The general and logical theory of automata. In Collected Works, A. H. Taub, Ed. Vol. 5. Pergamon Press, Oxford, England, 288-328.

Wolfram, S. 2002. A New Kind of Science. Wolfram Media, Champaign, IL.

Young, S., Alfke, P., Fewer, C., McMillian, S., Blodget, B., And Levi, D. 2003. A high i/o reconfigurable crossbar switch. In IEEE Symposium on Field-Programmable Custom Computing Machines. IEEE CS, IEEE Press, Napa, CA, 3-10. 